\title{
BIM Maturity Index: Analysis and Comparison of Architecture Office's BIM Performance in Porto Alegre
}

\author{
Monika Maria Stumpp \\ Universidade Federal do Rio Grande do Sul | Brasil | monistumpp@hotmail.com \\ Rodrigo Vitória Alves \\ Universidade Federal do Rio Grande do Sul | Brasil | rodrigoalves98@gmail.com \\ Carlo Rossano Manica \\ Universidade Federal do Rio Grande do Sul | Brasil | crmanica@gmail.com
}

\begin{abstract}
This study evaluated the BIM Maturity Levels of architecture offices in Porto Alegre (Brazil) by adapting the method developed by Succar (2009). It was carried out by applying a questionary in face-to-face interviews and converting the qualitative data obtained into quantitative, ultimately generating the BIM Maturity Index: a percentage number that can be classified and compared. The preliminary results indicate a significant discrepancy in BIM competences between the case studies while reasserting challenges faced by them in a regional scope: the scarcity of external stakeholders appropriately inserted on the BIM process and the undersupplied BIM knowledge offered in academic courses.
\end{abstract}

Keywords: BIM; BIM Maturity; Project Managing; Case studies; Architecture offices.

\section{INTRODUÇÃO}

Apesar dos estudos desenvolvidos na área, tanto o número de empresas que utilizam a tecnologia BIM (Building Information Modeling ou Building Information Model Modelagem da Informação da Construção ou Modelo da Informação da Construção) quanto seu ensino em universidades brasileiras são inferiores aos verificados em países europeus e nos EUA. Como apontado por Ruschel et all. (2013) em estudo sobre o ensino do BIM no Brasil, poucos profissionais brasileiros se encontram habilitados para utilizar o conceito em sua plena função.

Ponderando o exposto no parágrafo anterior e tendo em vista a dificuldade de utilização do BIM em escala nacional, este artigo analisa como o BIM vem sendo utilizado por empresas brasileiras de arquitetura na cidade de Porto Alegre, no estado do Rio Grande do Sul, Brasil (RS).

A contribuição por ele trazida reside em responder os seguintes questionamentos: (a) os escritórios de arquitetura empregam a plataforma BIM? (b) qual o grau de conhecimento dos escritórios relativo às funcionalidades e ao potencial do BIM?

O presente artigo transpõe para a realidade portoalegrense o método desenvolvido pelo pesquisador Bilal Succar (idealizador da BIMelnniative), a fim de obter o grau de maturidade BIM dentre as empresas da capital gaúcha e a consequente composição de um quadro de maturidade BIM de escritórios de arquitetura portoalegrenses. O escopo é vislumbrar a situação real vivida por esses escritórios e a percepção de seus líderes no que se refere à implementação do BIM. Pretende-se, portanto, contribuir com os escritórios de Arquitetura na superação de barreiras e no embasamento da decisão de adotar ou não o BIM, entendendo que esta abordagem pode oferecer algumas soluções tanto para o aumento de qualidade e produtividade de serviços, quanto para a redução de desperdícios e custos.

\section{CONTEXTUALIZAÇÃO}

No Brasil, o BIM começou a ser introduzido no início dos anos 2000 e foi conquistando espaço em várias áreas, como a acadêmica. No entanto, a transição dos sistemas CAD (Computer Aided Design) para a plataforma BIM enfrenta dificuldades. As adversidades que se contrapõem ao pleno uso do BIM resultam, principalmente, da incompreensão geral das formas de integração desse sistema com o processo de projeto (Manzione, 2013).

Segundo Nardeli (2018), na última década "várias empresas enfrentaram o desafio de gerenciar centenas de empreendimentos concomitantes a distâncias continentais entre si, sujeitos a diferentes legislações urbanas e normas locais para licenciamento de projetos, bem como realidades diversas de custos de material e mão de obra, compondo, sem dúvida, um complexo cenário de governança e gestão". Neste contexto, o BIM passou a ser visto como um recurso essencial, unindo esforços dos setores público e privado para sua disseminação pelo país. Esforço este que resultou na publicação de um decreto que instituiu a Estratégia Nacional de Disseminação do Building Information Modelling no Brasil - Estratégia BIM BR (Decreto 9.377/ 2018) (Brasil, 2018). O sucesso desta lei depende, porém, da "qualidade da gestão pública e da 
dinâmica futura e grau de maturidade nesta matéria já alcançada pelo setor". (Nardelli, 2018).

Passados quase vinte anos da introdução do BIM, os resultados ainda revelam-se modestos, quando se considera o volume produzido pela indústria da construção civil brasileira. Neste sentido, cabe o questionamento: seria possivel avaliar o grau de maturidade alcançado até aqui pelo setor em seu esforço de implantação do BIM no Brasil? (Nardelli e Tonso, 2014).

A metodologia de Succar (Succar et all, 2012) vem ao encontro deste questionamento. Succar (2009) criou uma metodologia que analisa, quantifica e qualifica o uso do BIM em setores, organizações e empreendimentos, considerando oito variáveis: objetivos, fases e marcos; publicações de referência; diretrizes estabelecidas e casos de sucessos; normas técnicas; marco regulatório; indicadores de desempenho; sistemas de formação e capacitação; infraestrutura tecnológica. Com base nesses indicadores, é possível estabelecer uma escala de graus de maturidade em cinco níveis, do inicial ao otimizado, tema a seguir desenvolvido.

\section{MATURIDADE EM BIM}

Modelos de maturidade de capacidade, como o proposto por Succar (Succar et all, 2012) identificam um conjunto de melhorias, de processos que permitem aos implementadores alcançarem benefícios significativos para os negócios. Essa metodologia mostra-se pertinente para estudos de caso, pois apresenta a vantagem de propor uma avaliação do grau de maturidade de organizações na adoção e no desempenho do BIM em seus processos, operações e relações com outras organizações. Por estabelecer parâmetros para mensurar os estágios de maturidade, permite não apenas avaliar o status da organização, mas também visualizar passos futuros que levem a níveis mais altos de maturidade e, com isso, a um processo de permanente melhora no desempenho. Destaca-se que Succar desenvolve essa metodologia para fazer face a dificuldades enfrentadas por organizações que, em algum momento, enxergam o BIM e/ou suas ferramentas como boas alternativas para o desenvolvimento de seus trabalhos e optam por implementá-las.

A metodologia consolida-se na Matriz de Maturidade BIM (ou BIM), que tem dois eixos: conjuntos de capacidade em BIM e índice de maturidade. As tabelas que guiam a avaliação dos requisitos estão divididas em quatro partes: tecnologia, processos, políticas e estágios. A parte referente à tecnologia analisa o desempenho e as relações com o processo BIM de três quesitos: software (usos e os produtos extraídos do software, bem como sua inserção ou não nas estratégias da empresa), hardware (equipamentos) e rede (armazenamento e compartilhamento). Os processos englobam recursos (infraestrutura física e de conhecimento); atividades e fluxo de trabalho (papéis, habilidades e dinâmicas); produtos e serviços (entregáveis); liderança. As políticas abrangem três quesitos: preparatória (treinamento, transferência de conhecimento); regulatória (códigos, regulamentações e padrões); contratual. Os estágios referem-se aos definidos pelo método da BIMelnnitiave, sendo três no total. As escalas (divididas em micro, meso e macro) referem-se à área de abrangência dos processos em BIM da empresa, ligados à presença ou não da multidisciplinaridade com agentes externos.

A matriz é criada por um sistema de pontos. Ela tem como resultado um índice percentual capaz de comparar e classificar certo grupo utilizador da ferramenta BIM. Esse índice indica o quão avançado está o avaliado no processo BIM, obtendo-se a possibilidade de classificação em diferentes níveis de maturidade, definidos em ordem crescente de progressão como (Succar, 2009): maturidade a (inicial) - as ferramentas de BIM foram implantadas (software de modelagem ou outros), porém não existe uma estratégia geral e os processos e políticas do BIM não estão definidos; maturidade b (definido) - os agentes seniores orientam o uso do BIM, existe documentação de processos e políticas, bem como manuais de treinamento, guias e padrões de entrega, possibilidades do mercado ainda não são exploradas; maturidade c (gerenciado) - a empresa tem objetivos claros, com planos de ação e monitoramento, a ideia do BIM é compartilhada com todos os integrantes da equipe, o marketing aproveita as oportunidades do BIM; maturidade d (integrado) - funções e metas para o BIM são parte da organização, o BIM é utilizado para atrair e manter clientes, existe boa colaboração com os parceiros e as entregas de projeto (modelo) são sincronizadas, a produtividade é previsível, o sistema de gerenciamento da qualidade é associado aos padrões do BIM e a metas de desempenho; maturidade e (otimizado) - proatividade para alterações de processos ou políticas, soluções inovadoras de produtos, processos e oportunidades de negócios são procuradas e seguidas de forma implacável, são constantemente revisados os usos de softwares, existe revisão periódica dos padrões de entrega, utilizando novas funcionalidades dos softwares e chegando a melhorias dos produtos e da produtividade.

Os cinco níveis de maturidade BIM são pontuados, do inicial, com 0 (zero) ponto, ao otimizado, com 40 (quarenta) pontos. A progressão de níveis mais baixos para níveis mais altos indica melhorias no controle do processo BIM, evidenciando também melhorias na previsibilidade dos resultados, através da minimização de variações nas competências, performances e custos.

Exemplo de utilização do nível de maturidade Bim no Brasil é encontrado em Catelani (2019). Segundo o autor, em 2017 o nível de maturidade BIM do Brasil, para o componente da Liderança, poderia ser considerado médiobaixo, que é descrito como: 'há um ou mais líderes voluntários e/ou impulsionadores BIM informais atuando no país'. "Os principais esforços de disseminação do BIM nesse período eram realizados por desenvolvedores de softwares e por algumas empresas, organizações ou grupos específicos e isolados". Em 2018, quando foi desenvolvida a 'Estratégia BIM BR', o país evoluiu para o nível de 'média maturidade', que é descrito como: 'há um grupo de trabalho ou comitê unificado dirigindo a implementação / difusão de BIM no país'. (Catelani, 2019).

\section{METODOLOGIA}

Para alcançar os objetivos foram realizados pesquisa teórica, estudos de casos e análise (Fig. 1). A pesquisa teórica aborda dois enfoques: o uso do BIM no Brasil (Garrido et all, 2015; Cornetet e Florio, 2015; Barison e Santos, 2013; Garbini e Brandão, 2013; Oliveira e Pereira, 2011; Souza et all., 2009) e a estrutura conceitual de Bilal 
Succar (Succar 2009, 2012). Os conceitos desenvolvidos pelo pesquisador Bilal Succar, fundamentam-se nos textos do próprio autor, distribuídos em meios acadêmicos e de forma on-line. Foram também examinados autores que estudaram a estrutura teórica de Succar ou que nela se apoiaram para desenvolver seus trabalhos (Rodrigues, 2018).

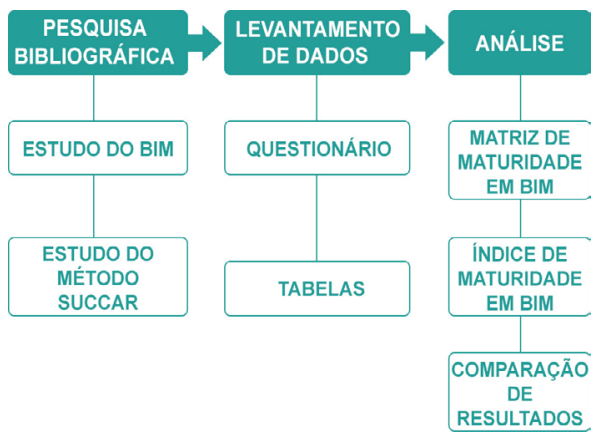

Figura 1: Gráfico esquemático do processo metodológico

\section{ESTUDOS DE CASO}

Os estudos de caso compreendem as seguintes etapas: seleção dos escritórios; elaboração de questionário; aplicação do questionário em entrevistas; análise de dados; elaboração das conclusões.

O questionário faz parte da entrevista semiestruturada elaborada por Rodrigues (2018), tendo como base a metodologia de Succar, estando dividido em cinco áreas: perfil da empresa e a transição para o BIM; tecnologia; processos e políticas; previsibilidade e variabilidade; metas e objetivos BIM da empresa.

As entrevistas foram realizadas nos próprios escritórios, com pelo menos um colaborador dentre sócios, coordenadores de projetos ou gerentes do BIM. Foram selecionados e convidados a participar escritórios clientes da Autodesk e Graphisoft. Aceito o convite, o agendamento das entrevistas foi formalizado por meio de cartas enviadas aos escritórios.

Segundo a classificação brasileira de porte de estabelecimentos por número de empregados, grande parte dos escritórios entrevistados constituem microempresas (até nove colaboradores). Os estudos de caso 3, 6 e 7 abrangem pequenas empresas (10 a 49 colaboradores). 0 escritório 7 é o maior no que se refere ao número de colaboradores: cerca de 20 pessoas.

\section{ANÁLISE}

A forma como os dados foram analisados e transferidos para a matriz seguiu a metodologia proposta por Succar $(2009,2012)$ e adotada por Rodrigues (2018) em estudo realizado com escritórios brasileiros. $\mathrm{Na}$ análise, procedeuse a transformação das respostas obtidas na entrevista em valores numéricos, utilizando a Matriz de Maturidade BIM, para gerar o índice de Maturidade BIM. Com a obtenção do Índice, é possível classificar o estudo de caso (nível inicial a otimizado) utilizando-se a tabela do Grau de Maturidade BIM (Fig. 2) e apurando o nível de maturidade. É importante salientar que os níveis de maturidade servem tanto para classificar o Índice de Maturidade BIM de cada estudo, quanto os Conjuntos de Capacidade BIM e suas subdivisões encontrados na Matriz de Maturidade BIM (Fig. 3). Nesse caso cada nível abrange um intervalo de pontuação e uma definição de acordo com cada quesito da avaliação. A correlação entre as definições e as pontuações de cada nível é o que transforma os dados qualitativos em quantitativos.

\begin{tabular}{|c|c|c|c|}
\hline \multicolumn{4}{|c|}{ GRAU DE MATURIDADE BIM } \\
\hline & $\begin{array}{c}\text { Nível de } \\
\text { Maturidade }\end{array}$ & $\begin{array}{c}\text { Classificação } \\
\text { Textual }\end{array}$ & $\begin{array}{c}\text { Classificação } \\
\text { Numeral }\end{array}$ \\
\hline a & Inicial & $\begin{array}{c}\text { Baixa } \\
\text { maturidade }\end{array}$ & $0-19 \%$ \\
\hline b & Definido & $\begin{array}{c}\text { Média-baixa } \\
\text { maturidade }\end{array}$ & $20-39 \%$ \\
\hline c & Gerenciado & $\begin{array}{c}\text { Média } \\
\text { maturidade }\end{array}$ & $40-59 \%$ \\
\hline d & Integrado & $\begin{array}{c}\text { Média-alta } \\
\text { maturidade }\end{array}$ & $60-79 \%$ \\
\hline e & Otimizado & $\begin{array}{c}\text { Alta } \\
\text { maturidade }\end{array}$ & $80-100 \%$ \\
\hline
\end{tabular}

Figura 2: Grau de Maturidade em BIM - Adaptado de Silvério (2018)

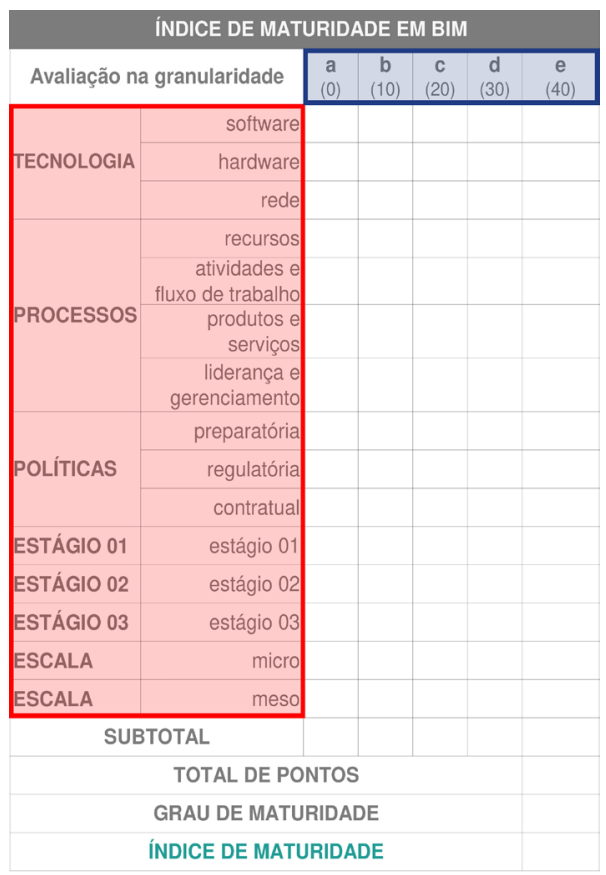

CONJUNTO DE CAPACIDADES E SUAS SUBDIVISÕES

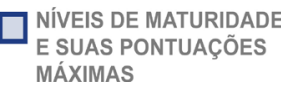
MÁXIMAS
Figura 3: A Matriz de Maturidade em BIM 


\section{RESULTADOS}

Até o presente momento, foram realizados sete estudos de caso (EC), dentre os previstos, apresentados, a seguir, na ordem com que as entrevistas foram realizadas.

\section{ESTUDO DE CASO 1}

O escritório do primeiro estudo de caso foi fundado em 2008. Trabalha atualmente com duas principais linhas tipológicas: o ramo imobiliário e os projetos autorais, que englobam os concursos de arquitetura. Além disso, considera uma terceira linha tipológica que surgiu com a implementação do BIM: a coordenação projetos em BIM para outras empresas e instituições. No processo de implantação do BIM, optou pelo Archicad e realizaram-se dois projetos pilotos. Não houve desenvolvimento prévio de templates e bibliotecas, sendo eles feitos concomitantemente à produção de projetos. O investimento inicial foi considerado baixo, sendo o hardware julgado como o elemento de maior custo. Observa-se, na Matriz de Maturidade (Fig. 4), que, na maioria dos aspectos, a pontuação atinge o nível integrado. No estágio 1, a pontuação alcança inteiramente o nível otimizado, o que revela haver constante revisão de tecnologias, processos e políticas BIM, buscando inovação e altos níveis de desempenho. O primeiro estudo de caso atinge um Índice de Maturidade em BIM de 70,31\%, classificando-se no nível Integrado (média-alta maturidade).

\section{ÍNDICE DE MATURIDADE EM BIM (ESTUDO DE CASO 01)}

\begin{tabular}{|c|c|c|c|c|c|c|}
\hline \multicolumn{2}{|c|}{ Avaliação na granularidade } & $\begin{array}{l}a \\
(0)\end{array}$ & $\begin{array}{c}\mathbf{b} \\
(10)\end{array}$ & $\begin{array}{c}\mathbf{c} \\
(20)\end{array}$ & $\begin{array}{c}d \\
(30)\end{array}$ & $\begin{array}{c}\mathbf{e} \\
(40)\end{array}$ \\
\hline \multirow{3}{*}{ TECNOLOGIA } & software & & & & 30 & \\
\hline & hardware & & & & 25 & \\
\hline & rede & & & & 30 & \\
\hline \multirow{4}{*}{ PROCESSOS } & recursos & & & & & 35 \\
\hline & $\begin{array}{r}\text { atividades e } \\
\text { fluxo de trabalho }\end{array}$ & & & & 30 & \\
\hline & $\begin{array}{r}\text { produtos e } \\
\text { serviços }\end{array}$ & & & & & 35 \\
\hline & $\begin{array}{r}\text { liderança e } \\
\text { gerenciamento }\end{array}$ & & & & 30 & \\
\hline \multirow{3}{*}{ POLÍTICAS } & preparatória & & & & 25 & \\
\hline & regulatória & & & & 30 & \\
\hline & contratual & & & & 30 & \\
\hline ESTÁGIO 01 & estágio 01 & & & & 30 & \\
\hline ESTÁGIO 02 & estágio 02 & & & & & 40 \\
\hline ESTÁGIO 03 & estágio 03 & & & 20 & & \\
\hline ESCALA & micro & & & & 30 & \\
\hline ESCALA & meso & & & & 30 & \\
\hline \multicolumn{2}{|c|}{ SUBTOTAL } & 0 & 0 & 20 & 320 & 110 \\
\hline \multicolumn{6}{|c|}{ TOTAL DE PONTOS } & 450 \\
\hline \multicolumn{6}{|c|}{ GRAU DE MATURIDADE } & 28,13 \\
\hline \multicolumn{6}{|c|}{ ÍNDICE DE MATURIDADE } & $70,31 \%$ \\
\hline
\end{tabular}

Figura 4: Índice de Maturidade em BIM do estudo de caso 01

\section{ESTUDO DE CASO 2}

O escritório correspondente ao segundo estudo de caso iniciou suas atividades no ano de 2004. Atualmente, dedica-se a edifícios residenciais unifamiliares e prédios comerciais. De acordo com o entrevistado, o perfil dos clientes projeta-se cada vez mais às incorporadoras. A implementação do BIM ocorreu em 2014 e o software escolhido foi o Archicad. Nesse processo, não houve consultorias, mas uma avaliação informal de prós e contras. Houve um projeto-piloto e o desenvolvimento de templates e bibliotecas ocorreu gradualmente, durante a produção. O investimento inicial no BIM foi considerado médio, sendo o custo em software considerado o mais elevado. Pela análise de sua matriz de maturidade em BIM (Fig. 5), observam-se pontuações máximas nos recursos (processos) e na escala meso. Isto significa que os fatores físicos do ambiente de trabalho são revisados para garantir a satisfação pessoal e a produtividade, assim como indica níveis altos de colaboração nos projetos, que são realizados por equipes interdisciplinares, incluindo a maioria das partes interessadas. Com um Índice de $47,66 \%$, o estudo de caso número 2 alcança o nível de maturidade Gerenciado (média maturidade).

INDICE DE MATURIDADE EM BIM (ESTUDO DE CASO 02)

\begin{tabular}{|c|c|c|c|c|c|c|}
\hline \multicolumn{2}{|c|}{ Avaliação na granularidade } & $\begin{array}{l}a \\
(0)\end{array}$ & $\begin{array}{c}\mathbf{b} \\
(10)\end{array}$ & $\begin{array}{c}\text { c } \\
(20)\end{array}$ & $\begin{array}{c}d \\
(30)\end{array}$ & $\begin{array}{c}\mathrm{e} \\
(40)\end{array}$ \\
\hline \multirow{3}{*}{ TECNOLOGIA } & software & & & 20 & & \\
\hline & hardware & & 10 & & & \\
\hline & rede & & & & 25 & \\
\hline \multirow{4}{*}{ PROCESSOS } & recursos & & & & & 40 \\
\hline & $\begin{array}{r}\text { atividades } e \\
\text { fluxo de trabalho }\end{array}$ & & & & 30 & \\
\hline & $\begin{array}{r}\text { produtos e } \\
\text { serviços }\end{array}$ & & & 20 & & \\
\hline & $\begin{array}{r}\text { liderança e } \\
\text { gerenciamento }\end{array}$ & & & 20 & & \\
\hline \multirow{3}{*}{ POLITICAS } & preparatória & & & 20 & & \\
\hline & regulatória & & & 20 & & \\
\hline & contratual & & 10 & & & \\
\hline ESTÁGIO 01 & estágio 01 & & & & 30 & \\
\hline ESTÁGIO 02 & estágio 02 & & 10 & & & \\
\hline ESTÁGIO 03 & estágio 03 & & 10 & & & \\
\hline ESCALA & micro & & & 20 & & \\
\hline ESCALA & meso & & & 20 & & \\
\hline \multicolumn{2}{|c|}{ SUBTOTAL } & 0 & 40 & 140 & 85 & 40 \\
\hline \multicolumn{6}{|c|}{ TOTAL DE PONTOS } & 305 \\
\hline \multicolumn{6}{|c|}{ GRAU DE MATURIDADE } & 19,06 \\
\hline \multicolumn{6}{|c|}{ ÍNDICE DE MATURIDADE } & $47,66 \%$ \\
\hline
\end{tabular}

Figura 5: Índice de Maturidade em BIM do estudo de caso 02

\section{ESTUDO DE CASO 3}

O escritório avaliado no terceiro estudo de caso inicia sua inserção no mercado através do ramo de maquetes eletrônicas em 2007, inaugurando sua produção arquitetônica de fato em 2009. Ele constitui o estudo de caso de maior porte dentre os entrevistados, com cerca de 20 colaboradores. As tipologias comerciais e residenciais representam as principais linhas de atuação da empresa, além de produção para concursos e alguma atuação em arquitetura de interiores. A transição para a plataforma BIM contou com treinamentos, consultorias e projeto-piloto, além da criação gradual de um manual BIM para uso interno e disponibilização de cursos online para novos contratados. O investimento inicial foi considerado muito alto, sendo a compra de licenças considerada como o elemento mais custoso do processo de transição, o 
software escolhido foi o Revit (Autodesk). A matriz de maturidade (Fig. 6) evidencia que a maioria dos valores permanece nos níveis integrado e otimizado, atingindo pontuação máxima em cinco quesitos. Isso indica que o escritório se alinha fortemente a estratégias que ligam o processo de produção arquitetônica em BIM com práticas de revisão de desempenho e produtividade, colaboração interdisciplinar, padronização e documentação de soluções ligadas ao processo e às metas BIM da empresa. O terceiro estudo de caso encerra a avaliação com um índice de $77,34 \%$, portando atingindo nível de maturidade Integrado.

\section{ÍNDICE DE MATURIDADE EM BIM (ESTUDO DE CASO 03)}

\begin{tabular}{|c|c|c|c|c|c|c|}
\hline \multicolumn{2}{|c|}{ Avaliação na granularidade } & \multirow[t]{2}{*}{$\begin{array}{c}a \\
(0)\end{array}$} & \multirow[t]{2}{*}{$\begin{array}{c}\mathbf{b} \\
(10)\end{array}$} & \multirow[t]{2}{*}{$\begin{array}{c}\mathbf{c} \\
(20)\end{array}$} & \multirow[t]{2}{*}{$\begin{array}{c}\text { d } \\
(30)\end{array}$} & \multirow{2}{*}{$\begin{array}{c}\mathrm{e} \\
(40) \\
35\end{array}$} \\
\hline \multirow{3}{*}{ TECNOLOGIA } & software & & & & & \\
\hline & hardware & & & 15 & & \\
\hline & rede & & & & 30 & \\
\hline \multirow{4}{*}{ PROCESSOS } & recursos & & & & & 40 \\
\hline & $\begin{array}{r}\text { atividades e } \\
\text { fluxo de trabalho }\end{array}$ & & & & & 40 \\
\hline & $\begin{array}{r}\text { produtos e } \\
\text { serviços }\end{array}$ & & & & & 35 \\
\hline & $\begin{array}{r}\text { liderança e } \\
\text { gerenciamento }\end{array}$ & & & & 30 & \\
\hline \multirow{3}{*}{ POLITICAS } & preparatória & & & & 30 & \\
\hline & regulatória & & & & 25 & \\
\hline & contratual & & & & 30 & \\
\hline ESTÁGIO 01 & estágio 01 & & & & & 40 \\
\hline ESTÁGIO 02 & estágio 02 & & & & & 40 \\
\hline ESTÁGIO 03 & estágio 03 & & & & & 40 \\
\hline ESCALA & micro & & & & & 35 \\
\hline ESCALA & meso & & & & 30 & \\
\hline \multicolumn{2}{|c|}{ SUBTOTAL } & 0 & 0 & 15 & 175 & 305 \\
\hline \multicolumn{6}{|c|}{ TOTAL DE PONTOS } & 495 \\
\hline \multicolumn{6}{|c|}{ GRAU DE MATURIDADE } & 30,94 \\
\hline \multicolumn{6}{|c|}{ ÍNDICE DE MATURIDADE } & $77,34 \%$ \\
\hline
\end{tabular}

Figura 6: Índice de Maturidade em BIM do estudo de caso 03

\section{ESTUDO DE CASO 4}

O quarto escritório investigado surgiu do trabalho de duas arquitetas que começaram a projetar durante o período acadêmico utilizando o CAD, sendo a transição para o BIM feita em 2009. A empresa foi constituída oficialmente em 2011 e hoje se dedica a projetos de arquitetura de interiores de pequeno porte para clientes de classe média e média alta. A transição para o BIM ocorreu quando o escritório ainda não estava formalizado, as arquitetas faziam trabalhos de forma autônoma, contando com seu conhecimento BIM adquirido individualmente, sem uma avaliação mais aprofundada de prós e contras. Dessa forma, para a escolha do software as sócias fizeram cursos para aprendizado de Revit e Archicad, sendo feita a opção pelo Archicad. Os templates e as bibliotecas iniciais utilizados foram os originais do software, com adições e criações ao longo do tempo. Não houveram retornos ao $C A D$, apenas para exportação a terceiros, que foi argumentado como sendo algo ainda necessário, visto a pouca disponibilidade de agentes externos inseridos ao BIM. O software foi considerado como o elemento mais custoso da transição BIM, sendo o investimento inicial visto como de alto custo. Observa-se que, em todos os aspectos, a pontuação atinge niveis entre o definido e o integrado (Fig. 7), sendo que sua pontuação máxima se encontra no quesito contratual, da seção de Políticas, o que indica que a organização está alinhada através da confiança e dependência mútua, indo além das barreiras contratuais. Seu índice de Maturidade em BIM é de $40,63 \%$, classificando o estudo no nível Gerenciado.

\section{ÍNDICE DE MATURIDADE EM BIM (ESTUDO DE CASO 04)}

\begin{tabular}{|c|c|c|c|c|c|c|}
\hline \multicolumn{2}{|c|}{ Avaliação na granularidade } & $\begin{array}{l}\mathbf{a} \\
(0)\end{array}$ & $\begin{array}{c}\text { b } \\
(10)\end{array}$ & $\begin{array}{c}\mathbf{c} \\
(20)\end{array}$ & $\begin{array}{c}d \\
(30)\end{array}$ & $\begin{array}{c}\mathbf{e} \\
(40)\end{array}$ \\
\hline \multirow{3}{*}{ TECNOLOGIA } & software & & & 20 & & \\
\hline & hardware & & 10 & & & \\
\hline & rede & & & 20 & & \\
\hline \multirow{4}{*}{ PROCESSOS } & recursos & & & 20 & & \\
\hline & $\begin{array}{r}\text { atividades e } \\
\text { fluxo de trabalho }\end{array}$ & & & 20 & & \\
\hline & $\begin{array}{r}\text { produtos e } \\
\text { serviços }\end{array}$ & & & 15 & & \\
\hline & $\begin{array}{r}\text { liderança e } \\
\text { gerenciamento }\end{array}$ & & & 15 & & \\
\hline \multirow{3}{*}{ POLÍTICAS } & preparatória & & 10 & & & \\
\hline & regulatória & & 10 & & & \\
\hline & contratual & & & & 30 & \\
\hline ESTÁGIO 01 & estágio 01 & & & 15 & & \\
\hline ESTÁGIO 02 & estágio 02 & & & 20 & & \\
\hline ESTÁGIO 03 & estágio 03 & & & 20 & & \\
\hline ESCALA & micro & & & 15 & & \\
\hline ESCALA & meso & & & 20 & & \\
\hline \multicolumn{2}{|c|}{ SUBTOTAL } & 0 & 30 & 200 & 30 & 0 \\
\hline \multicolumn{6}{|c|}{ TOTAL DE PONTOS } & 260 \\
\hline \multicolumn{6}{|c|}{ GRAU DE MATURIDADE } & 16,25 \\
\hline \multicolumn{6}{|c|}{ ÍNDICE DE MATURIDADE } & $40,63 \%$ \\
\hline
\end{tabular}

Figura 7: Índice de Maturidade em BIM do estudo de caso 04

\section{ESTUDO DE CASO 5}

O escritório visualizado no quinto estudo de caso iniciou suas atividades em 2004. Hoje ele é reconhecido no mercado por sua produção em BIM, por já ter realizado alguns projetos importantes em âmbito regional. Sua principal frente de atuação é a produção para o mercado imobiliário de incorporadoras, sendo as tipologias residencial, comercial, de uso misto ou institucional as mais usuais. A introdução do BIM ocorreu em 2010, com opção pelo software Archicad. Durante a transição, os custos com hardware e software evidenciaram-se como os mais altos. Houve projeto-piloto durante a transição BIM; os templates e as bibliotecas foram feitos gradualmente pelo escritório, conforme surgia a necessidade de novos elementos. Através de sua Matriz de Maturidade (Fig. 8), observa-se que os valores mais altos se referem a processos, estágios e escalas. No estágio 3 - integração baseada em rede -, foi atingida a pontuação máxima, o que indica que a integração dos modelos e dos fluxos de trabalho é continuamente revista e otimizada. As novas eficiências, alinhamentos, e os resultados são ativamente perseguidos por uma equipe de projeto interdisciplinar firmemente unida. Os modelos integrados contribuem para muitos agentes envolvidos ao longo da cadeia produtiva. Com um Índice de Maturidade de $69,53 \%$ o quinto estudo de caso se classifica no nível Integrado. 
INDICE DE MATURIDADE EM BIM (ESTUDO DE CASO 05)

\begin{tabular}{|c|c|c|c|c|c|c|}
\hline \multicolumn{2}{|c|}{ Avaliação na granularidade } & \multirow[t]{2}{*}{$\begin{array}{l}a \\
(0)\end{array}$} & \multirow[t]{2}{*}{$\begin{array}{c}\text { b } \\
(10)\end{array}$} & \multirow[t]{2}{*}{$\underset{(20)}{\mathbf{C}}$} & \multirow{2}{*}{$\begin{array}{c}\begin{array}{c}\mathbf{d} \\
(30)\end{array} \\
30\end{array}$} & \multirow[t]{2}{*}{$\begin{array}{c}\text { e } \\
(40)\end{array}$} \\
\hline \multirow{3}{*}{ TECNOLOGIA } & software & & & & & \\
\hline & hardware & & & & 30 & \\
\hline & rede & & & 20 & & \\
\hline \multirow{4}{*}{ PROCESSOS } & recursos & & & & 30 & \\
\hline & $\begin{array}{r}\text { atividades e } \\
\text { fluxo de trabalho }\end{array}$ & & & & & 35 \\
\hline & $\begin{array}{r}\text { produtos e } \\
\text { serviços }\end{array}$ & & & & & 35 \\
\hline & $\begin{array}{r}\text { liderança e } \\
\text { gerenciamento }\end{array}$ & & & 20 & & \\
\hline \multirow{3}{*}{ POLÍTICAS } & preparatória & & & & 30 & \\
\hline & regulatória & & & 20 & & \\
\hline & contratual & & & 20 & & \\
\hline ESTÁGIO 01 & estágio 01 & & & & 30 & \\
\hline ESTÁGIO 02 & estágio 02 & & & & & 35 \\
\hline ESTÁGIO 03 & estágio 03 & & & & & 40 \\
\hline ESCALA & micro & & & & & 35 \\
\hline ESCALA & meso & & & & & 35 \\
\hline \multicolumn{2}{|c|}{ SUBTOTAL } & 0 & 0 & 80 & 150 & 215 \\
\hline \multicolumn{6}{|c|}{ TOTAL DE PONTOS } & 445 \\
\hline \multicolumn{6}{|c|}{ GRAU DE MATURIDADE } & 27,81 \\
\hline \multicolumn{6}{|c|}{ ÍNDICE DE MATURIDADE } & $69,53 \%$ \\
\hline
\end{tabular}

Figura 8: Índice de Maturidade em BIM do estudo de caso 05

\section{ESTUDO DE CASO 6}

O arquiteto fundador do sexto escritório observado começou seus trabalhos em 1984. No entanto, o escritório com o nome e a sociedade como atualmente é conhecido foi inaugurado em 2012. Este é um estudo de caso particularmente interessante, pois, tendo começado a atuar na década de 80 , testemunhou várias transformações tecnológicas, desde o modus operandi com papel e lapiseira, passando pelo $\mathrm{CAD}$, até chegar, nos dias de hoje, ao BIM. As tipologias mais realizadas pelo escritório são prédios residenciais, comerciais e mistos, voltados para o mercado imobiliário, tendo como principais clientes as incorporadoras. A transição para o BIM começou em 2014, tendo como principal motivo o desejo de aumento do domínio das fases preliminares do projeto, visando principalmente a diminuição do retrabalho. Houve a realização de dois cursos para o aprendizado do software Archicad e nenhum projeto-piloto. O entrevistado comenta que não houveram retornos ao CAD. Consideram a transição para o BIM como sendo de custo médio. $\mathrm{Na}$ matriz de maturidade BIM (Fig. 9), nota-se que os valores mais baixos se encontram nas políticas, indicando carência nos treinamentos, principalmente falta de alinhamento a possíveis estratégias da empresa. Observam-se limitações na documentação e na padronização da forma como a empresa utiliza o BIM. O estudo atinge um Índice de $\mathbf{5 0 , 0 0 \%}$, portanto, alcançando nível Gerenciado.
INDICE DE MATURIDADE EM BIM (ESTUDO DE CASO 06)

\begin{tabular}{|c|c|c|c|c|c|c|}
\hline \multicolumn{2}{|c|}{ Avaliação na granularidade } & $\begin{array}{c}a \\
(0)\end{array}$ & $\begin{array}{c}\mathbf{b} \\
(10)\end{array}$ & $\begin{array}{c}\text { C } \\
(20)\end{array}$ & $\begin{array}{c}\text { d } \\
(30)\end{array}$ & $\begin{array}{c}\mathbf{e} \\
(40)\end{array}$ \\
\hline \multirow{3}{*}{ TECNOLOGIA } & software & & & 15 & & \\
\hline & hardware & & & 15 & & \\
\hline & rede & & & & 30 & \\
\hline \multirow{4}{*}{ PROCESSOS } & recursos & & & 20 & & \\
\hline & $\begin{array}{r}\text { atividades e } \\
\text { fluxo de trabalho }\end{array}$ & & & & 25 & \\
\hline & $\begin{array}{r}\text { produtos e } \\
\text { serviços }\end{array}$ & & & 20 & & \\
\hline & $\begin{array}{r}\text { liderança e } \\
\text { gerenciamento }\end{array}$ & & & & 25 & \\
\hline \multirow{3}{*}{ POLÍTICAS } & preparatória & & 10 & & & \\
\hline & regulatória & & 10 & & & \\
\hline & contratual & & & 20 & & \\
\hline ESTÁGIO 01 & estágio 01 & & & & 30 & \\
\hline ESTÁGIO 02 & estágio 02 & & & & 30 & \\
\hline ESTÁGIO 03 & estágio 03 & & & 20 & & \\
\hline ESCALA & micro & & & & 30 & \\
\hline ESCALA & meso & & & 20 & & \\
\hline \multicolumn{2}{|c|}{ SUBTOTAL } & 0 & 20 & 130 & 170 & 0 \\
\hline \multicolumn{6}{|c|}{ TOTAL DE PONTOS } & 320 \\
\hline \multicolumn{6}{|c|}{ GRAU DE MATURIDADE } & 20,00 \\
\hline \multicolumn{6}{|c|}{ ÍNDICE DE MATURIDADE } & $50,00 \%$ \\
\hline
\end{tabular}

Figura 9: Índice de Maturidade em BIM do estudo de caso 06

\section{ESTUDO DE CASO 7}

O sétimo escritório abordado foi fundado em 1985 como uma marcenaria, paulatinamente mudando seu foco para projetos de arquitetura efêmera. Por volta do ano 2000 , voltou-se inteiramente para a produção arquitetônica de edificações residenciais - uni e multifamiliares - e comerciais. A implementação do BIM ocorreu em 2011, utilizando o Archicad. Houve treinamento e consultoria. Durante a transição, o escritório dividiu-se em grupos estratégicos para a criação de templates, bibliotecas e remodelagem de projetos antigos feitos em CAD. Na Figura 6, observa-se que as maiores pontuações estão em produtos e serviços. Isto indica que a empresa extrai uma gama satisfatória de produtos BIM, como quantitativos, cronogramas, orçamentos etc. Atinge parcialmente o nível otimizado no estágio 3 , demonstrando que a integração de modelos e fluxos de trabalho é continuamente revista e otimizada. Os valores alcançados por estes estudos de caso estão na tabela abaixo (Fig. 10). O índice de $\mathbf{5 0 , 0 0 \%}$ do último estudo de caso configura nível de maturidade Integrado. 
ÍNDICE DE MATURIDADE EM BIM (ESTUDO DE CASO 07)

\begin{tabular}{|c|c|c|c|c|c|c|}
\hline \multicolumn{2}{|c|}{ Avaliação na granularidade } & $\begin{array}{l}\mathbf{a} \\
(0)\end{array}$ & $\begin{array}{c}\mathbf{b} \\
(10)\end{array}$ & $\begin{array}{c}\text { c } \\
(20)\end{array}$ & $\underset{(30)}{\mathbf{d}}$ & $\begin{array}{c}\mathbf{e} \\
(40)\end{array}$ \\
\hline \multirow{3}{*}{ TECNOLOGIA } & software & & & & 30 & \\
\hline & hardware & & & & 30 & \\
\hline & rede & & & & 30 & \\
\hline \multirow{4}{*}{ PROCESSOS } & recursos & & & 20 & & \\
\hline & $\begin{array}{l}\text { atividades e } \\
\text { fluxo de trabalho }\end{array}$ & & & & 30 & \\
\hline & $\begin{array}{r}\text { produtos e } \\
\text { serviços }\end{array}$ & & & & & 35 \\
\hline & $\begin{array}{r}\text { liderança e } \\
\text { gerenciamento }\end{array}$ & & & & 25 & \\
\hline \multirow{3}{*}{ POLÍTICAS } & preparatória & & & 15 & & \\
\hline & regulatória & & & & 25 & \\
\hline & contratual & & & & 30 & \\
\hline ESTÁGIO 01 & estágio 01 & & & & 30 & \\
\hline ESTÁGIO 02 & estágio 02 & & & & 30 & \\
\hline ESTÁGIO 03 & estágio 03 & & & 20 & & \\
\hline ESCALA & micro & & & & & 35 \\
\hline ESCALA & meso & & & 20 & & \\
\hline \multicolumn{2}{|c|}{ SUBTOTAL } & 0 & 0 & 75 & 260 & 70 \\
\hline \multicolumn{6}{|c|}{ TOTAL DE PONTOS } & 405 \\
\hline \multicolumn{6}{|c|}{ GRAU DE MATURIDADE } & 25,31 \\
\hline \multicolumn{6}{|c|}{ ÍNDICE DE MATURIDADE } & $63,28 \%$ \\
\hline
\end{tabular}

Figura 10: Índice de Maturidade em BIM do estudo de caso 07

\section{ANÁLISE}

Os estudos de caso 1, 3, 5 e 7 configuram os maiores índices de maturidade, atingindo o nível Integrado. Os casos 3 e 7 passaram por processos de transição BIM parecidos: além de consultorias e treinamentos, houve a criação de projetos-piloto, bibliotecas e templates de forma bem definida e integrada às estratégias de implantação. No entanto, há entre eles uma diferença de 14\% no índice de maturidade BIM, que se explica principalmente nos processos, nos estágios e nas escalas. Pode-se aferir que, no estudo de caso 3 , houve mais acompanhamento de desempenho e revisão dos processos BIM, bem como o estabelecimento melhor definido de parcerias BIM com terceiros envolvidos no processo de projeto e/ou execução.

Os estudos de caso 2, 4 e 6 possuem índices de maturidade menores do que $60 \%$ e maiores do que $39 \%$, o que os classifica no nível gerenciado. Verificam-se algumas similaridades, principalmente no que tange ao processo de transição para o BIM: todos eles passaram por processos informais de transição, sem consultorias ou treinamentos abrangentes; a criação de templates e bibliotecas ocorreu paulatinamente, conforme a necessidade, sem uma estratégia predefinida; não houve projetos-piloto bem definidos ou de alguma forma alinhados à estratégia de implantação. De modo geral, observa-se, nesses estudos de caso, uma abordagem que ainda tende à ênfase na modelagem, apesar de haver avanços nos fatores processuais e políticos do BIM e de a visão BIM ser compartilhada por toda a equipe. Há neles um distanciamento maior entre as metas BIM da empresa com o que realmente se faz possível na prática do que os outros estudos de caso.
Mostra-se possível traçar características e condições semelhantes em todos os estudos de caso. Por exemplo, com a transição para o BIM, houve aumento significativo da fase preliminar de projeto, devido principalmente à grande quantidade de informações que devem ser fornecidas na etapa inicial de modelagem. Isto induz à resolução de grande parte do projeto nessa fase e, por consequência, a diminuição do tempo gasto nas fases finais, como projeto executivo, documentação, orçamentos e etc, corroborando com o exposto pela Curva MacLeamy, que indica como funciona o fluxo de trabalho em BIM em contraste com o fluxo de trabalho tradicional (Fig. 11).

Nesse artigo, a Curva MacLeamy embasa e avigora as verificações feitas através dos relatos coletados junto aos estudos de caso com relação ao tempo destinado nas fases do projeto e a transição do sistema CAD para o BIM (MacLeamy, 2010).
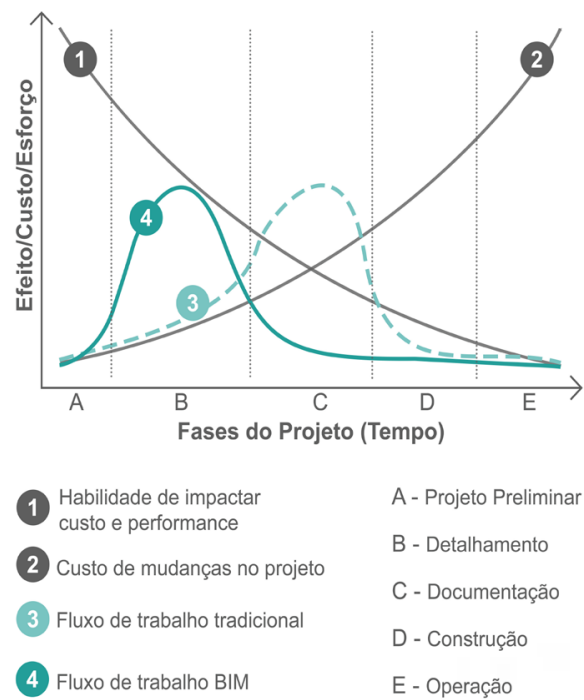

Habilidade de impacta custo e performance

(2)

Custo de mudanças no projeto

Fluxo de trabalho tradicional

Fluxo de trabalho BIM

A - Projeto Preliminar

B - Detalhamento

C - Documentação

D - Construção

E - Operação

Figura 11: Gráfico conceitual que ilustra a Curva MacLeamy adaptado de MacLeamy (2010)

Apesar disso e da grande maioria dos entrevistados considerar a utilização do BIM como um fator de competitividade no mercado, todos eles relataram que não houve aumento no valor dos honorários pela utilização da plataforma.

É interessante observar que nenhum dos Índices de Maturidade BIM dos estudos de caso alcançou o nível Otimizado, principalmente pelo fato de que a pontuação para atingir níveis otimizados depende diretamente de uma rede ampla de agentes externos inseridos ao BIM que não condiz com a realidade regional, como observado pelos relatos dos escritórios, nem com o contexto brasileiro ( Ruschel et all., 2013), principalmente se compararmos com os Estados Unidos e países europeus (Fig. 12). 


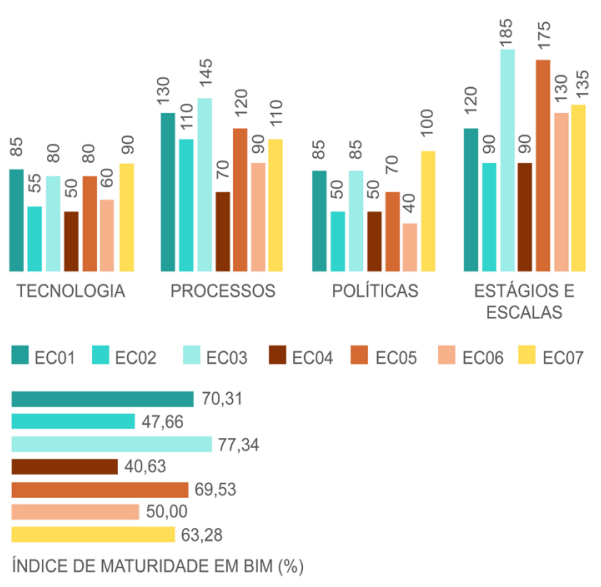

Figura 12: Comparação de resultados (pontuação por área e índice de maturidade em BIM de cada estudo de caso)

\section{CONCLUSÃO}

Em consonância com os resultados preliminares, concluise que, mesmo existindo disparidade entre os escritórios nas competências referentes ao BIM, alguns desafios são enfrentados por todos eles. Por conseguinte, acentuam-se as dificuldades em comum a serem transpostas no cenário regional e nacional: pouca disponibilidade de terceiros inseridos ao processo BIM, principalmente na área de projetos complementares; falta de conhecimento da totalidade da plataforma por agentes da esfera AEC; escassez de disciplinas nas universidades que tratem sobre $o$ assunto. Observa-se também que existem fatores internos a serem trabalhados para que se elevem as capacidades referentes ao desempenho BIM de empresas, instituições e usuários da plataforma, principalmente no tocante à conscientização de que a plataforma BIM envolve fatores processuais e políticos os quais podem ser aprimorados.

Pode-se observar que a disparidade dos valores dos Índices de Maturidade entre os estudos de caso se dá principalmente pela forma como a transição para o BIM foi feita e como ela foi manejada ao longo do tempo. Os estudos de caso que fizeram transições mais aprofundadas, com avaliações, consultorias, treinamentos e que continuam avaliando e aperfeiçoando os processos e as políticas BIM dentro de seus escritórios apresentaram maior valor de Maturidade BIM. Além disso, é importante levar em consideração que algumas barreiras a serem transpostas a níveis regionais e nacionais também tem relação com a performance dos níveis de maturidade BIM, tais como a pouca disponibilidade de terceiros apropriadamente inseridos ao processo BIM, principalmente na área de projetos complementares; falta de conhecimento da totalidade da plataforma por agentes da esfera AEC; escassez de disciplinas nas universidades que tratem sobre $o$ assunto.

\section{AGRADECIMENTOS}

Exemplo: Agradecemos aos escritórios participantes e a FAPERGS-UFRGS pela bolsa de pesquisa.

\section{REFERÊNCIAS}

BRASIL (2018). Decreto n. 9.377, de 17 de maio de 2018. Institui a Estratégia de Disseminação do Building Information Modelling. Diário Oficial da União, Brasília, Edição 95, Seção 1, p. 3. Atos do Poder Executivo.Brasil.

Barison, E.M. \& Santos, E.T. (2013). Atual cenário da implementação de BIM no mercado da construção civil da cidade de São Paulo e demanda por especialistas. In SCHEER, S. et all. Modelagem da informação da construção: uma experiência brasileira em BIM. Curitiba: Capes.554p.

Catelani, W. (2019). Como avançar na maturidade BIM no Brasil? Artigo do especialista. Disponivel em https://cbic.org.br/artigodo-especialista-como-avancar-na-maturidade-bim-no-brasil/

Cornetet, B. \& W. Florio, W. (2015). Reflexão sobre a implantação do BIM em três escritórios de arquitetura em Porto Alegre, de 2010 a 2015. Encontro Brasileiro de Tecnologia de Informação e Comunicação na Construção, Recife.

Garbini, M. A. L. \& Brandão, D. Q. (2013). Implantação da tecnologia BIM analisada em quatro escritórios de arquitetura. Cadernos Proarq (2013), v. 21, pp. 125-146

Garrido, C. M. et all. (2015). Using BIM for Last Planner System: Case Studies in Brazil. International Workshop on Computing in Civil Engineering. pp. 604-611.

Manzione, L. (2013). Proposição de uma estrutura conceitual para a gestão do processo de projeto colaborativo com o uso do BIM. Tese de Doutorado - Escola Politécnica. São Paulo, 353 $\mathrm{p}$

MacLeamy, P. (2010). The Future of the Building Industry (3/5): The Effort Curv. Disponivel em https://www.youtube.com/watch?v=9bUIBYc_GI4

Nardelli, E.S. (2018). BIM and Public Bidding in Brazil. Proceedings of the 22th Conference of the Iberoamerican Society of Digital Graphics, pp. 1212-1219, São Paulo.

Nardelli, E. S. \& Tonso L. G. (2014). BIM - Barreiras institucionais para a sua implantação no Brasil. Proceedings of the 18th Conference of the Iberoamerican Society of Digital Graphics (2014), pp. 408-411, Uruguay - Montevideo. Disponível em http://papers.cumincad.org/cgibin/works/Show?sigradi2014 292.

Oliveira, L. \& Pereira, A. (2011). Mudanças metodológicas decorrentes da implantação recente de BIM em escritórios de arquitetura", in Proceedings of the 15th Conference of the Iberoamerican Society of Digital Graphics, Santa Fé, Argentina.

Rodrigues, A. (2018). Grau de Maturidade BIM: Estudos de Caso em empresas projetistas de Arquitetura na cidade de São Paulo", Monografia (Especialização em Gestão de Projetos na Construção) - Escola Politécnica da Universidade de São Paulo. São Paulo, 164 pp.

Ruschel, R.C. et all (2013). O ensino de BIM no Brasil: onde estamos? Ambiente Construído 13 (2), 151-165.

Souza, L.L.A. de, Amorim, S.R.L. \& Lyrio, A. de M. (2009). Impactos do uso do Bim em escritórios de arquitetura: oportunidades no mercado Imobiliário. Gestão \& Tecnologia de Projetos, 4 (2), p.26-53.

Succar, B. (2009). Building Information Modelling Maturity Matrix Research on Building Information Modelling and Construction Informatics: Concepts and Technologies Australia, p.65-103. Austrália: IGI Publishing, nov. 2010.

Succar, B. W. \& Williams, S. A. (2012). Measuring BIM performance: Five metrics. Architectural Engineering and Design Management, Volume 8, pp. 120-142. School of Architecture and Built Environment, University of Newcastle, Australia. 